\title{
Follow-up study on management of chronic obstructive pulmonary disease in Hunan Province, the People's Republic of China
}

This article was published in the following Dove Press journal:

International Journal of COPD

10 May 2016

Number of times this article has been viewed

\section{Yuqin Zeng ${ }^{1, *}$ \\ Jianxian $\mathrm{Xu}^{1, *}$ \\ Shan Cai' \\ Fen Jiang' \\ Anmei $\mathrm{Hu}^{2}$ \\ Huayun $\mathrm{Liu}^{3}$ \\ Chengli Bei ${ }^{4}$ \\ Ping Chen'}

'Department of Respiratory Medicine, The Second Xiangya Hospital, Central South University, Changsha, Hunan,

${ }^{2}$ Department of Respiratory Medicine, Shenzhen City Baoan District Central Hospital, Shenzhen, Guangdong, ${ }^{3}$ Department of Respiratory Medicine, The Yueyang First People's Hospital, Yueyang, Hunan, ${ }^{4}$ Department of Respiratory Medicine, Changsha Central Hospital, Changsha,

People's Republic of China

*These authors contributed equally to this work
Correspondence: Shan Cai Department of Respiratory Medicine, The Second Xiangya Hospital, Central South University, I 39 Middle Renmin Road, Changsha, Hunan Province, People's Republic of China Tel +86 I 3875858644 Fax +8673 I 82246096 Email caishan70@sina.com
Background: In 2009, our study showed an extreme imbalance and disparity in COPD-related health resources allocation at three levels of public hospitals (PHs) in Hunan Province of the People's Republic of China, especially in second-level PHs. Moreover, most Chinese citizens accept their health care services in first- and second-level PHs for economic and geographical reasons, as well as because of the incomplete transfer system in the health care services. To improve diagnosis and treatment ability of pulmonologists in second-level PHs, an intervention that provided training combined with spirometry equipment was carried out in three PHs from six second-level PHs. The aim of this follow-up study was to evaluate the changes associated with COPD-related health resources allocation and the effect of the intervention 4 years later. Methods: The data regarding the availability of spirometers, inhalation agents for COPD, and COPD-related health care education for local residents were collected from 57 PHs in 2009 and $48 \mathrm{PHs}$ in 2013. Pulmonologists working in these PHs were asked to complete a questionnaire individually. Six second-level PHs (three in the intervention group and the other three in the control group [without training and spirometry equipment]) that further took part in the survey in 2009 were reevaluated to determine the doubtful diagnostic ratio and the confirmation ratio of COPD. The differences between 2009 and 2013 data was analyzed.

Results: A total of 762 questionnaires were completed. Compared with 2009, spirometerequipped ratio raised from $40 \%$ to $69 \%$ in $2013(P=0.03)$. The overall inhalation agent-equipped ratio increased from $58 \%$ in 2009 to $88 \%$ in $2013(P=0.001)$. The total rate of health education raised from $35 \%$ in 2009 to $63 \%$ in $2013(P=0.04)$. In total, 204 pulmonologists from six PHs (selected for study in 2009) completed the questionnaires in 2013. The median score of COPD knowledge questionnaire in the intervention group was higher than that of the control group ( 80 vs 50 , respectively, $P<0.01$ ). Further, the clinical doubtful diagnostic ratio $(15 \%)$ and the diagnosis ratio of COPD $(3 \%)$ in the intervention group were higher than in the control group ( $4 \%$ and $1 \%$, respectively, $P<0.05$ ) even after 4 years.

Conclusion: COPD-related health resources allocation improved in the 4 years following intervention at three levels of PHs in the People's Republic of China. Short-term training combined with spirometry equipment had a sustained effect on improving the physicians' understanding and diagnosis ability of COPD.

Keywords: COPD, public hospitals, pulmonologists, training, China

\section{Introduction}

COPD is a common preventable and treatable disease that has been a growing health problem, and remains a major consumer of health care resources in the world. ${ }^{1}$ Unfortunately, a majority of studies from the general population and primary care 
have reported that a high proportion of individuals fulfilling the COPD diagnosis criteria remain undiagnosed., ${ }^{2,3}$

In the People's Republic of China, all public hospitals (PHs) are ranked at three levels (first-, second-, and third-level hospitals, higher levels are better) by the Ministry of Health of the local and central bureau according to the physical size of the medical campus, the number of inpatient beds, the type of medical equipment, etc. ${ }^{4}$ Generally, higher level PHs are built in bigger cities, which lead to a huge disparity in health care resources and services between cities and rural areas. ${ }^{5}$ As in our previous study, we found an extreme imbalance and disparity in spirometry equipment, availability of inhaler agents, and COPD-related health care education at three levels of PHs in Hunan Province of the People's Republic of China, especially in the first- and second-level PHs. ${ }^{6}$ Moreover, a serious shortage of medical specialists exists in rural areas because most specialists preferred to work in high-level hospitals located in urban areas. ${ }^{7}$ In addition, the educational level of the majority of physicians in rural areas ranges from 3 to 5 years of medical college after secondary school training. ${ }^{6}$ Most physicians who are unfamiliar with COPD guidelines will have difficulties understanding the treatment options, outcomes, and prognosis of COPD. ${ }^{8,9}$ Therefore, improving the physicians' diagnosis and treatment ability of diseases is urgent.

Providing continued medical education to physicians can be effective in improving their psychomotor skills and application of knowledge. ${ }^{10}$ In the People's Republic of China, learning in a top-level hospital is an efficient way of continuing medical education for rural doctors who want to improve their knowledge of medicine. Even so, not all rural doctors have chances to continue their medical education in a top-level hospital. Similar to the findings of our previous study, we found that short-term training combined with spirometry equipment could improve physicians' knowledge about COPD, including the ability to diagnose COPD and assess its severity. ${ }^{11}$

With a rapid growth in the Chinese economy and deepening reforms in the Chinese health care system, questions about imbalance and disparity in the COPD-related health resources allocation arise, and we wondered if the intervention was still working. The aim of this follow-up study was to evaluate the changes of COPD-related health resources allocation and the effect of the intervention 4 years later.

\section{Materials and methods}

\section{Participants and trial design}

The Second Xiangya Hospital of Central South University institutional review board approved the study. Written informed consent was obtained from all the participants.
A clustering-stratified random sampling, cross-sectional study was used to investigate the COPD-related health resources allocation at three levels of PHs in Hunan Province, the People's Republic of China, in 2009. At the same time, a questionnaire was sent to pulmonologists in those PHs, including questions about baseline characteristics (literacy and professional title) and knowledge about COPD. In 2013, we collected data from the same PHs to investigate the COPD-related health resources allocation and sent the same questionnaire to pulmonologists in those PHs to survey their knowledge about COPD.

In addition, we used a randomized parallel-group controlled design to compare the effects of an intervention in 2009, including a 1-hour training course and supplying spirometry equipment, with a control that did not receive the intervention.

Six second-level PHs without spirometers were randomized using a random number generator and allocated to treatment vs control groups at a ratio of $1: 1$.

Diagnosis and treatment level of pulmonologists in those PHs was calculated according to the referred medical records in the same 3 -month period before the intervention (October 1, 2008 to December 31, 2008), during (January 1, 2009 to March 31, 2009), and at the follow-up visit (January 1, 2013 to March 31, 2013).

\section{Survey questionnaire design}

Two questionnaires were used in this study. One design included hospital levels, investigating whether their wards had spirometers, types of inhaled bronchodilator medications used, and whether COPD-related health education was provided for patients or not. The other questionnaire aimed at investigating the physicians' knowledge about COPD and was devised by a pulmonary expert advisory group, which was based on the existing published recommendations of the Chinese COPD guidelines. ${ }^{12}$ The questionnaire intended to test the COPD knowledge was designed to investigate the demographic information, professional titles, and educational levels of pulmonologists, and to test their knowledge of COPD diagnosis and treatment based on the COPD guidelines. In total, ten single-choice questions were presented including main manifestations of COPD, pathogenesis of COPD, diagnostic criteria, evaluation of pulmonary function, guideline criteria for severity grading, therapy for COPD including pharmacotherapy and oxygen, the common causes of acute exacerbation COPD, and indications for noninvasive ventilation. Every question was worth 10 points. Not answered and wrong answers were given a 0 score. The reliability and validity of the questionnaire had been tested 
before. Briefly, Cronbach's alpha score measuring the internal consistency of questions was 0.73 and 0.77 for the first and the second questionnaires, respectively. ${ }^{6}$

The questionnaires were administered by a research assistant and surveyed anonymously. All pulmonologists participated by completing the questionnaires by themselves.

\section{Outcomes}

\section{Allocation of COPD-related health care resources}

Spirometer-equipped ratio, inhalation agent-equipped ratio, and availability ratio of COPD-related health care education at three levels of PHs in 2009 and 2013 were collected.

\section{Pulmonologists' basic knowledge about COPD}

Scores of the questionnaire investigating the COPD knowledge of pulmonologists were compared among the pulmonologists working at different levels of PHs in 2009 and 2013. Scores based on education levels or professional titles of different pulmonologists were calculated in 2013.

\section{Effect of short-term training}

Pulmonologists' doubtful diagnosis ratio and confirmation diagnosis ratio of COPD were calculated in six secondlevel PHs in the intervention group and the control group. Consistent with the ease of data collection for the study, we only collected data on all hospitalized patients over the age of 40 years from the referred medical records in both groups to compare the pulmonologists' clinical doubtful diagnostic ratio and diagnosis ratio of COPD. "Clinical doubtful diagnosis ratio" was defined as the number of hospitalized patients with clinical doubtful diagnosis of COPD/total number of hospitalized patients over the age of $40 \times 100 \%$; "diagnosis ratio of COPD" was defined as the number of hospitalized patients diagnosed with COPD/total number of hospitalized patients over the age of $40 \times 100 \%$.

\section{Data analysis}

All data were input and analyzed by using Statistical Product and Service Solutions 17.0 (SPSS 17.0) software. Chi-square test was used to compare results about spirometer-equipped ratio, inhalation agent-equipped ratio, availability rate of health education, clinical doubtful diagnostic ratio of COPD, diagnosis ratio of COPD, pulmonologists' education levels and professional titles, and accuracy of COPD questionnaires. Differences between the groups were considered significant if $P \leq 0.05$. Comparison of pulmonologists' scores was performed using a nonparametric test (Wilcoxon). The Mann-Whitney $U$ method was used to compare two groups, whereas the Kruskal-Wallis $H$ method was used for multiple groups. Spearman's rank correlation method was used to acquire multivariate linear regression analysis of pulmonologists' scores.

\section{Results}

\section{Baseline characteristics}

In 2009, there were 44 third-level PHs, 292 second-level PHs, and 193 first-level PHs. We randomized 57 PHs at the three different levels. In 2013, there were 48 PHs because nine first-level PHs were removed, six of which had changed their hospital levels and three had incomplete data. In 2009, questionnaires were sent to 893 pulmonologists in $57 \mathrm{PHs}$, and 869 were returned, with a recovery rate of $97 \%$. Only 846 questionnaires were effective according to the study design, resulting in an effective rate of 95\%. In 2013, 792 questionnaires were sent to pulmonologists in $48 \mathrm{PHs}$, and 776 questionnaires were returned, with a recovery rate of $98 \%$. Only 762 questionnaires were effective and fourteen were excluded because of any unanswered question, resulting in an effective rate of $98 \%$. The baseline characteristics of PHs and pulmonologists in 2009 and 2013 are shown in Table 1.

\section{Changes associated with COPD-related health resources allocation over the 4 years} Allocation of spirometers

The total spirometer-equipped ratio in 2013 was 69\%, and $0 \%, 75 \%$, and $100 \%$ for the first-, second-, and third-level PHs, respectively. Significant differences between the levels of PHs in 2013 were still seen among the spirometerequipped ratios $\left(\chi^{2}=27.05, P<0.0001\right)$. Compared with the condition in 2009 , the equipped ratio was higher in the second-level PHs in $2013\left(\chi^{2}=8.39, P=0.003\right)$. There was no change in the first- and third-level PHs between the 2 years $(P>0.05)$ (Table 2).

\section{Allocation of inhalation agents}

The total inhalation agent-equipped ratio in 2013 was $88 \%$, and $33 \%, 100 \%$, and $100 \%$ for the first-, second-, and third-level PHs, respectively. Significant differences were observed among the inhaled agent-equipped ratios at different levels of PHs in $2013\left(\chi^{2}=29.71, P<0.0001\right)$. Significant difference regarding equipped ratio was observed in the second-level PHs between 2009 and 2013 $\left(\chi^{2}=8.39, P=0.004\right)$. There was no change in the thirdlevel PHs during the 4 years. No difference existed in the first- and third-level PHs between 2009 and $2013\left(\chi^{2}=1.29\right.$, $P=0.26$ ) (Table 2). 
Table I Baseline characteristics of public hospitals (PHs) and pulmonologists

\begin{tabular}{|c|c|c|c|c|c|c|c|c|}
\hline \multirow[t]{2}{*}{ Variable } & \multicolumn{2}{|l|}{ Total } & \multicolumn{2}{|c|}{ Third-level PHs } & \multicolumn{2}{|c|}{ Second-level PHs } & \multicolumn{2}{|c|}{ First-level PHs } \\
\hline & 2009 & 2013 & 2009 & 2013 & 2009 & 2013 & 2009 & 2013 \\
\hline $\mathrm{PHs}(\mathrm{n})$ & 57 & 48 & 15 & 15 & 24 & 24 & 18 & 9 \\
\hline Pulmonologists (n) & 846 & 762 & 192 & 204 & 559 & 506 & 95 & 52 \\
\hline \multicolumn{9}{|l|}{ Pulmonologists' } \\
\hline \multicolumn{9}{|l|}{ educational level (n) } \\
\hline High & 82 & 109 & 73 & 93 & 9 & 16 & 0 & 0 \\
\hline Medium & 604 & 520 & 117 & 111 & 424 & 380 & 63 & 29 \\
\hline Low & 160 & 133 & 2 & 0 & 126 & 110 & 32 & 23 \\
\hline \multicolumn{9}{|l|}{ Pulmonologists' } \\
\hline \multicolumn{9}{|l|}{ professional level (n) } \\
\hline Senior & 115 & 124 & 40 & 45 & 55 & 67 & 20 & 12 \\
\hline Intermediate & 378 & 332 & 100 & 98 & 244 & 205 & 34 & 29 \\
\hline Junior & 353 & 306 & 52 & 61 & 260 & 234 & 41 & 11 \\
\hline
\end{tabular}

Notes: Pulmonologists' educational level was categorized into three levels: high educational level means PhD and master's degree of medicine, median means bachelor's degree of medicine, and low indicates someone who had just completed junior medical college or medical school. Pulmonologists' professional level was categorized into three levels: senior title implies deputy chief physician or chief physician, intermediate title implies attending physician, and junior title implies resident physician.

Inhalation corticosteroids/long-acting beta agonists, long-acting anticholinergic medications, and short-acting beta agonists were equipped in second- and third-level PHs. However, there was only salbutamol aerosol (short-acting beta agonist) in first-level PHs.

\section{Allocation of COPD-related health care education}

The overall availability ratio of COPD-related health care education in 2013 was $63 \%$, and $22 \%, 63 \%$, and $87 \%$ for the first-, second-, and third-level PHs, respectively. Significant differences were still considered among the availability rates of COPD-related health care education at different levels of PHs in 2013 ( $\left.\chi^{2}=9.96, P=0.006\right)$, but no difference was found between the second- and third-level PHs $\left(\chi^{2}=2.66, P=0.10\right)$. COPD-related health care education had been increasingly available from 2009 to $2013\left(\chi^{2}=4.17, P=0.04\right)$ (Table 2).

\section{Pulmonologists' basic knowledge of COPD}

Score distribution of pulmonologists' questionnaire about COPD knowledge

In 2009, the total median score of the pulmonologists' questionnaire about COPD knowledge was 80 , and 25, 80, and 90 for the first-, second-, and third-level PHs, respectively. In 2013, the total median score was 70 and 40, 60, and 90 for the first-, second-, and third-level PHs, respectively. The total median score declined from 80 in 2009 to 70 in 2013, and it declined from 80 to 60 at second-level PHs $(P<0.001)$. While pulmonologists' median score at first-level PHs increased from 25 in 2009 to 40 in 2013 ( $U=621.50, P<0.001$ ), there was no change at third-level PHs between the years 2009 and $2013(P=0.634)$.

\section{Accuracy rate of every question of physicians}

There was a clear difference in the accuracy rate (AR) of physicians at the three levels of PHs. AR was much better in the third-level PHs than in the first- and second-level PHs. The chi-squared test was used to calculate the difference in AR among different groups. Except for question four, which showed no difference between first- and second-level PHs $(P>0.05)$, AR of the rest of the nine questions showed significant differences among the pulmonologists at all the three levels (Table 3 ).

In general, the higher the level of PHs the better was the pulmonologists' basic knowledge of COPD. Compared with

Table 2 Allocation of COPD-related health resources in three levels of public hospitals (PHs) in 2009 and 2013

\begin{tabular}{|c|c|c|c|c|c|c|c|c|}
\hline \multirow{3}{*}{$\begin{array}{l}\text { Allocation of } \\
\text { resources (\%) }\end{array}$} & \multicolumn{2}{|l|}{ Total } & \multicolumn{2}{|c|}{ Third-level PHs } & \multicolumn{2}{|c|}{ Second-level PHs } & \multicolumn{2}{|c|}{ First-level PHs } \\
\hline & 2009 & 2013 & 2009 & 2013 & 2009 & 2013 & 2009 & 2013 \\
\hline & $(n=57)$ & $(n=48)$ & $(n=15)$ & $(n=15)$ & $(n=24)$ & $(n=24)$ & $(n=18)$ & $(n=9)$ \\
\hline Spirometers & 40 & 69* & 100 & 100 & 33 & $75^{*}$ & 0 & 0 \\
\hline Inhalation agents & 58 & $88^{*}$ & 100 & 100 & 71 & $100 *$ & 6 & 33 \\
\hline COPD-related & 35 & $63^{*}$ & 67 & 87 & 42 & 63 & 0 & 22 \\
\hline
\end{tabular}

health care education

Notes: *Chi-square test was used to compare in 2009 and $2013, P<0.05$. 
Table 3 Accuracy rate (AR) of every question of physicians in three levels of public hospitals (PHs) in 2013

\begin{tabular}{|c|c|c|c|}
\hline \multirow[t]{2}{*}{ Questionnaire } & \multicolumn{3}{|l|}{ AR (\%) } \\
\hline & $\begin{array}{l}\text { Third-level PHs } \\
(n=204)\end{array}$ & $\begin{array}{l}\text { Second-level PHs } \\
(n=506)\end{array}$ & $\begin{array}{l}\text { First-level PHs } \\
(n=52)\end{array}$ \\
\hline I. Hallmark symptom of COPD & $86 *$ & $55^{*}$ & $40 *$ \\
\hline 2. Pathogen of COPD & $97^{*}$ & $83^{*}$ & $65^{*}$ \\
\hline 3. Criteria of airflow limitation & $91^{*}$ & $48^{*}$ & $21^{*}$ \\
\hline 4. Diagnostic tools of COPD & $100 *$ & $47^{*}$ & 40 \\
\hline 5. Grading criteria of COPD & $83^{*}$ & $43^{*}$ & $23^{*}$ \\
\hline 6. Medicine of COPD & $91 *$ & $65^{*}$ & $27^{*}$ \\
\hline 7. Oxygen therapy of COPD & $81^{*}$ & $51 *$ & $3 I^{*}$ \\
\hline 8. Treatment of COPD & $90^{*}$ & $66^{*}$ & $29 *$ \\
\hline 9. Acute exacerbation cause of COPD & $98^{*}$ & $93 *$ & $79 *$ \\
\hline 10. Indication of noninvasive venation & $83^{*}$ & $56 *$ & $40 *$ \\
\hline
\end{tabular}

Notes: *Chi-square test was used to compare between any of the two groups, $P<0.05$.

2009, there was no improvement in the pulmonologists' basic knowledge of COPD in $2013(P>0.5)$.

Comparison of scores based on educational levels of different pulmonologists in 2013

Among the total 762 pulmonologists, there were 133, 520, and 109 pulmonologists with low, medium, and high educational level, respectively, with a corresponding median score of 100,70 , and 40 . The Kruskal-Wallis $H$-test was used to analyze the pulmonologists' scores among different groups. Higher educational level of the pulmonologist corresponded with a higher score on the COPD knowledge questionnaire $(P<0.001)$ (Figure 1).

Comparison of scores based on professional titles of different pulmonologists in 2013

Among the total 762 pulmonologists, there were 124, 332, and 306 pulmonologists with senior, intermediate, and junior title, respectively, with a corresponding median score of 90, 70, and 60. The Kruskal-Wallis $H$-test was used to analyze the pulmonologists' scores among different groups. The higher the professional title of the pulmonologist, the higher was the score on the COPD knowledge questionnaire $(P<0.001)$ (Figure 2).

Above all, pulmonologists' understanding of COPD was associated with hospital level, educational background, and professional title. Spearman's rank correlation was used to calculate correlation analysis $\left(r_{1}=0.693, r_{2}=0.630, r_{3}=0.469\right.$; $P<0.001)$.

Effect of short-term training combined with spirometry equipment on COPD diagnosis among the second-level hospitals 4 years after intervention Pulmonologists' basic knowledge of COPD from six second-level PHs in 2013

In total, 204 questionnaires were sent to pulmonologists (78 pulmonologists in the intervention group and 126 in the control group) in six PHs, which took part in the further study, with a recovery rate of $100 \%$. The median score of the intervention group was 80 , and that of the control group was $50(P<0.001)$. Significant differences between the control group and the intervention group were observed among

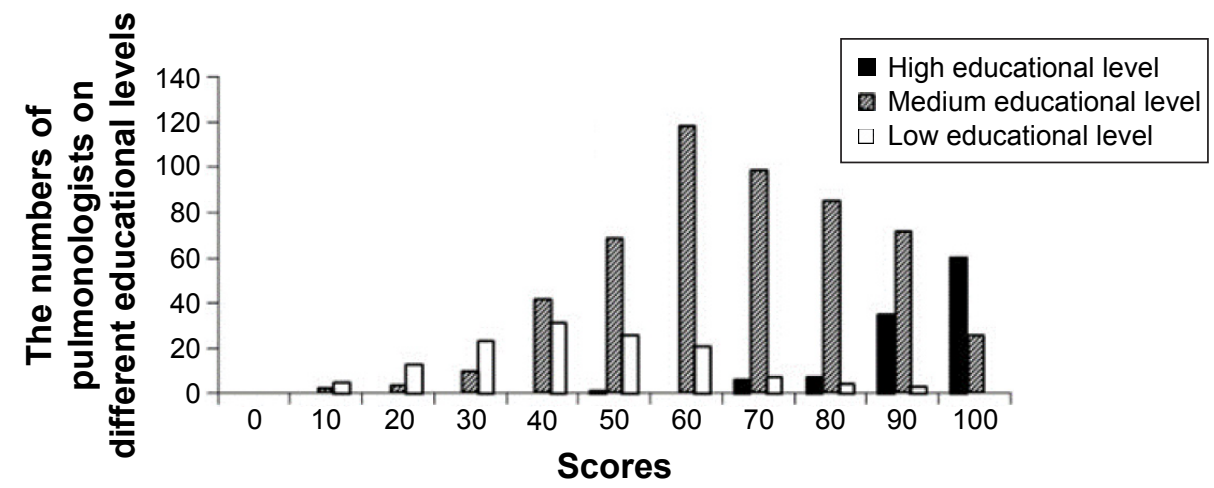

Figure I Scores of COPD knowledge questionnaire based on educational levels of different pulmonologists in 2013. 


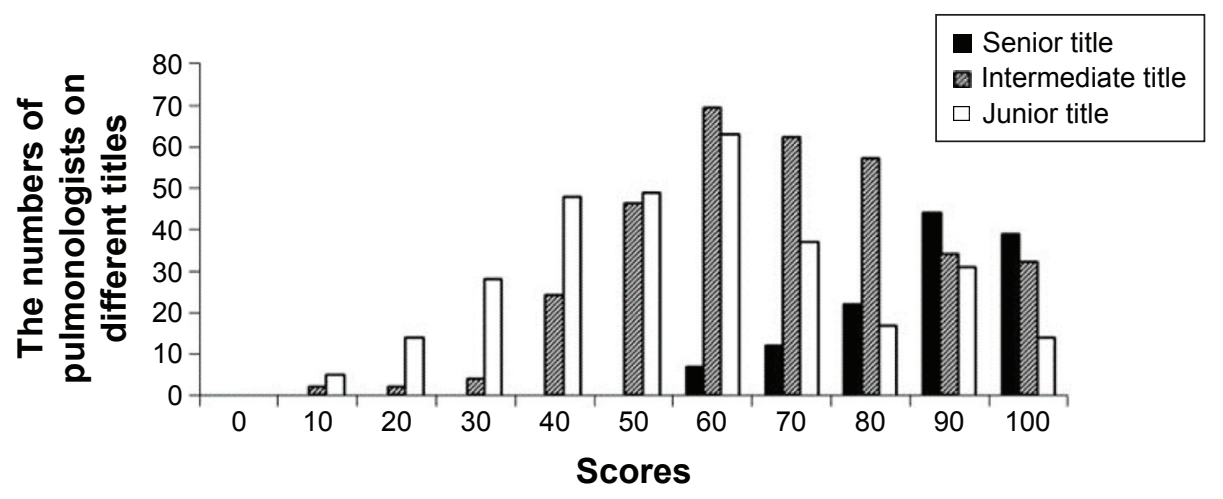

Figure 2 Scores of COPD knowledge questionnaire based on professional titles of different pulmonologists in 2013.

the median scores and AR (except question nine) in 2013 $(P<0.05)$ (Table 4).

\section{Comparison of pulmonologists' clinical doubtful diagnostic ratio and diagnosis ratio of COPD between the two groups during the three periods}

All of the hospitalized patients in our study were over the age of 40 years. During the period before intervention, there was no significant difference in the clinical doubtful diagnosis and diagnosis ratio of COPD between the control and the intervention groups. However, significant difference was found between the two groups during the intervention and follow-up visit $(P<0.001)$ (Table 5).

\section{Discussion}

Based on continuous renewal of Global Initiative for Chronic Obstructive Lung Disease (GOLD) guidelines, combined with the national conditions, the Chinese Society of pulmonologists formulated and updated the diagnosis and treatment guidelines for COPD, including definition, pathology and physiology, clinical manifestations, diagnosis, treatment, prevention, and other aspects of COPD. ${ }^{12}$ These guidelines made it easier for the Chinese pulmonologists to diagnose and treat COPD accurately and effectively. Even so, the COPDrelated health resources allocation, including the spirometry equipment, inhalation agents, COPD patient education, and even the pulmonologists' knowledge of COPD, are extremely imbalanced among the different levels of PHs in the People's Republic of China.

Spirometry is a safe, practical, and reproducible maximum breathing test that can be recommended as a "gold standard" to diagnose COPD and measure the severity of airflow obstruction. ${ }^{13}$ With the use of a spirometer, the diagnosis rate of COPD was doubled. ${ }^{14}$ Unfortunately, the allocation of spirometer resources is still imbalanced in South Central China, according to our study. At the third-level PHs, the spirometer equipment was sufficient, whereas at the first-level PHs, there were no spirometers even in 2013. For the second-level PHs,

Table 4 Accuracy rate (AR) of every question of physicians between the control group and the intervention group in six secondlevel $\mathrm{PHs}$

\begin{tabular}{|c|c|c|c|}
\hline \multirow[t]{3}{*}{ Questionnaire } & \multicolumn{2}{|l|}{ AR (\%) } & \multirow[t]{3}{*}{$P$-value } \\
\hline & Control group & Intervention group & \\
\hline & $(n=126)$ & $(n=78)$ & \\
\hline I. Hallmark symptom of COPD & 60 & 94 & $<0.00 I^{*}$ \\
\hline 2. Pathogen of COPD & 62 & 90 & $<0.00 I^{*}$ \\
\hline 3. Criteria of airflow limitation & 41 & 63 & $0.002 *$ \\
\hline 4. Diagnostic tools of COPD & 60 & 77 & $0.018 *$ \\
\hline 5. Grading criteria of COPD & 44 & 63 & $0.01 *$ \\
\hline 6. Medicine of COPD & 52 & 87 & $<0.001 *$ \\
\hline 7. Oxygen therapy of COPD & 40 & 62 & $0.002 *$ \\
\hline 8. Treatment of COPD & 57 & 88 & $<0.001 *$ \\
\hline 9. Acute exacerbation cause of COPD & 86 & 94 & 0.08 \\
\hline 10. Indication of noninvasive venation & 45 & 60 & $0.04 *$ \\
\hline
\end{tabular}

Notes: ${ }^{*}$ Chi-square test was used to compare between the control group and the intervention group, $P<0.05$. Abbreviation: $\mathrm{PHs}$, public hospitals. 
Table 5 Doubtful diagnosis and diagnosis ratio of COPD between the control group and the intervention group during three periods in six second-level PHs

\begin{tabular}{|c|c|c|c|c|c|c|c|c|c|}
\hline \multirow[t]{2}{*}{ Groups } & \multicolumn{3}{|c|}{ Before intervention } & \multicolumn{3}{|c|}{ During intervention } & \multicolumn{3}{|c|}{ Follow-up visit } \\
\hline & $\mathbf{n}$ & $\begin{array}{l}\text { Doubtful } \\
\text { diagnosis }\end{array}$ & Diagnosis & $\mathbf{n}$ & $\begin{array}{l}\text { Doubtful } \\
\text { diagnosis }\end{array}$ & Diagnosis & $\mathbf{n}$ & $\begin{array}{l}\text { Doubtful } \\
\text { diagnosis }\end{array}$ & Diagnosis \\
\hline Control group & 3,322 & $1 \%$ & $0 \%$ & 2,990 & $1 \%$ & $0 \%$ & 4,331 & $4 \%$ & $1 \%$ \\
\hline Intervention group & 2,867 & $1 \%$ & $0 \%$ & 2,930 & $14 \%$ & $3 \%$ & 4,136 & $15 \%$ & $3 \%$ \\
\hline$\chi^{2}$ & & 0.43 & & & 404.02 & 86.95 & & 303.44 & 79.50 \\
\hline$P$-value & & 0.51 & & & $<0.001$ & $<0.001$ & & $<0.001$ & $<0.001$ \\
\hline
\end{tabular}

Abbreviation: $\mathrm{PHs}$, public hospitals.

compared with the spirometer-equipped ratio in 2009, the ratio had risen in 2013. Lack of spirometry equipment may lead to underdiagnosis and misdiagnosis of COPD, which may explain the reason for a low diagnosis ratio of COPD in the People's Republic of China. Moreover, physicians who failed to realize the importance of spirometry in COPD management are mainly responsible for this condition..$^{15}$

Inhalation treatment was preferred for COPD patients. ${ }^{12}$ Our investigation showed that the total inhalation agentequipped ratio increased in 2013, especially for the secondlevel PHs. The increase indicates that more patients can receive inhaled treatment for COPD. However, further study showed that some of the second- and all of the first-level PHs were only equipped with salbutamol aerosol, which is inadequate for COPD treatment. Even though the Chinese government has made a great effort to make health care service more accessible and affordable to every person, cost is an important obstacle to the universality of inhaled medicine. In the People's Republic of China, most COPD patients are in rural areas and are economically disadvantaged. ${ }^{16} \mathrm{In}$ addition, age is another obstruction for the use of inhalers. Patients with COPD are generally elderly, and most elderly patients experience difficulties in achieving correct inhaler technique to gain optimum benefit from their inhaler even when in a stable clinical condition. ${ }^{17,18}$

Health education plays an important role in the whole process of COPD, including the diagnosis, treatment, and management of COPD. COPD-related health education can improve disease management skills, inhaler adherence, and patients' lifestyle choices, and decrease COPD-related emergency room visits and hospital admissions. Importantly, COPD-related health education positively influenced health-related quality of life. ${ }^{19,20}$ Our study found that the overall availability rate of COPD-related health care education had increased dramatically in 2013 compared with 2009, which means that more COPD patients were able to receive COPD-related education. The low availability of COPD-related health care education was due to the following reasons: 1) currently, the management of COPD patients has not been carried out effectively because of limited professional knowledge and a shortage of labor and funding. ${ }^{21}$ 2) Although most doctors know health education is important for COPD patients, many do not educate patients because they are busy treating patients..$^{22} 3$ ) Some patients cannot understand the contents of health education because of illiteracy, which is independently associated with poor health status. ${ }^{23}$ Overall, popularity of COPD-related health education demands the coordinate efforts of medical institutions, hospitals, medical workers, and the general public.

Pulmonologists' expertise and awareness of COPD guidelines are vital to the accurate diagnosis of COPD. ${ }^{24}$ The Chinese guidelines for the diagnosis and treatment of COPD have been revised and improved constantly over the past 2 decades..$^{12}$ However, implementation of these guidelines has been difficult, particularly in lower-level hospitals and rural areas. It was the same observance in our study: the total median score of the COPD knowledge questionnaire was even lower in 2013 than in 2009. In a further study, we had found the change of total score of the COPD knowledge questionnaire among groups was due to the proportion of the secondlevel PHs, which was a high proportion of the three levels PHs in our study, with lower total scores than in 2009. The study also showed that physicians' understanding of COPD was associated with hospital level, educational background, and professional title. In recent years, many second-level PHs underwent rapid expansion and had set up many new departments. Many of the expert clinicians were assigned to other departments, and the new respiratory clinicians were unfamiliar with COPD. This may be the main reason for low scores in the second-level PHs. Wilson et al discovered that persons with higher level of education possessed higher level of cognition, which may be the reason why those with higher educational level accept more new knowledge about COPD. ${ }^{25}$ In higher level PHs, pulmonologists had more chances to gain knowledge about COPD and study further: for example, by studying abroad, joining international or domestic academic exchanges, and continuing medicine education. By contrast, 
in lower-level PHs, shortage of staff, limitation of academic discipline, and insufficient funds hindered the burgeoning of continuing medical education, which lead to less knowledge about COPD for pulmonologists in these hospitals. ${ }^{26,27}$ Therefore, there is an urgent need for the pulmonologists and administrators to work together to improve knowledge about COPD. There are many interventions to improve physician application of knowledge, such as the use of multimedia, multiple instructional techniques, and multiple exposures. ${ }^{28}$ Ahmed et al analyzed 28 studies by the Johns Hopkins Evidence-based Practice Center and discovered that most demonstrated an improvement in knowledge after face-toface learning from specialists. ${ }^{29}$ Moreover, hands-on skill training showed great improvement in clinician skills. ${ }^{30}$ In the People's Republic of China, conductance of seminars in higher level PHs is a main method to enhance clinical skills. With the rapid development of the Internet and media, more network consultation systems are being carried out, and telemedicine is frequently used to deliver health care from a distance. Even so, challenges remain in the implementation of telemedicine in developing countries with immature systems as well as financial, health infrastructure, and legal and privacy issues. ${ }^{31}$ In our study, we found that with shorttime face-to-face education classes combined with spirometry equipment, pulmonologists' knowledge and diagnosis ability of COPD increased significantly and persisted for 4 years. This simple and effective method should be generalized at different levels of PHs, especially PHs without spirometry equipment in the People's Republic of China. More mature systems for further medical study need to be developed.

Our study has limitations. Only 48 PHs were investigated in 2013 compared with 57 PHs in 2009. Nine first-level PHs could not be included because of changing hospital levels and incomplete data, which may influence the evaluation of the hospital at this level. When calculating the effect of short-time training, we could not investigate the same physicians in the control group for before-and-after comparisons because of anonymity.

Several study strengths are worth noting. The numbers of investigated PHs and pulmonologists are sufficiently high. The PHs are at different levels and thus are representative of all of the PH levels in Hunan Province. The study reveals that the allocation of COPD-related health care resources has improved over time. It provides a frame of reference to our health administrators and policy-makers to manage health resources. Our short training program is simple and can be made available at many hospitals. It is persistently effective over time.

\section{Conclusion}

Although extreme imbalance and disparity still exist in COPD-related health resources allocation, such as spirometry equipment, inhaled agent availability, and COPD-related health care education, at three levels of PHs in South Central China, the situation has improved in the 4 years since the intervention began. Unfortunately, physicians' understanding of COPD declined, especially in the second-level PHs. The understanding was found to be associated with hospital level, educational background, and professional title. Overall, short-term training combined with spirometry equipment had a sustained effect on improving the physicians' understanding and diagnosis ability of COPD.

\section{Acknowledgments}

This study was supported by the Chronic Respiratory Disease Funds of the Chinese Medical Association (07010310039) and the National Key Clinical Specialty Construction Projects. We express thanks to all the physicians and patients who took part in this study.

\section{Disclosure}

The authors report no conflicts of interest in this work.

\section{References}

1. Goldcopd.org. [homepage on the Internet]. Global Strategy for Diagnosis, Management, and Prevention of COPD [updated January 2015; cited May 20, 2015]. Available from: http://www.goldcopd.org/. Accessed October 20, 2015.

2. Soriano JB, Ancochea J, Miravitlles M, et al. Recent trends in COPD prevalence in Spain: a repeated cross-sectional survey 1997-2007. Eur Respir J. 2010;36(4):758-765.

3. Bednarek M, Maciejewski J, Wozniak M, Kuca P, Zielinski J. Prevalence, severity and underdiagnosis of COPD in the primary care setting. Thorax. 2008;63(5):402-407.

4. Ministry of Health of the People's Republic of China Ministry of Health of the People's Republic of China. Annual Report on Health Statistics of China 2006 [in Chinese]. Beijing, China: Publishing House of the Peking Union Medical College; 2006.

5. Shi L. Health care in China: a rural-urban comparison after the socioeconomic reforms. Bull World Health Organ. 1993;71(6):723-736.

6. Bei $\mathrm{C}, \mathrm{Hu} \mathrm{A}, \mathrm{Liu} \mathrm{H}$, et al. Chronic obstructive pulmonary diseases related health resources allocation in Hunan Province of China. Iran J Public Health. 2013;42(6):543-551.

7. Wang C, Rao K, Wu S, Liu Q. Health care in China: improvement, challenges, and reform. Chest. 2013;143(2):524-531.

8. Desalu OO, Onyedum CC, Adeoti AO, et al. Guideline-based COPD management in a resource-limited setting - physicians' understanding, adherence and barriers: a cross-sectional survey of internal and family medicine hospital-based physicians in Nigeria. Prim Care Respir J. 2013;22(1):79-85.

9. Wong SS, Abdullah N, Abdullah A, et al. Unmet needs of patients with chronic obstructive pulmonary disease (COPD): a qualitative study on patients and doctors. BMC Fam Pract. 2014;15:67. 
10. O'Neil KM, Addrizzo-Harris DJ. Continuing medical education effect on physician knowledge application and psychomotor skills: effectiveness of continuing medical education: American College of Chest Physicians Evidence-Based Educational Guidelines. Chest. 2009; 135(Suppl 3):S37-S41.

11. Cai S, Qin L, Tanoue L, et al. Effects of one-hour training course and spirometry on the ability of physicians to diagnose and treat chronic obstructive pulmonary disease. PLoS One. 2015;10(2):e0117348.

12. Chronic Obstructive Pulmonary Disease Study Group, Thoracic Society, Chinese Medical Association (CMACOPDS). Guideline for diagnosis and treatment of COPD (revised edition 2007). Zhonghua Jie He He Hu Xi Za Zhi. 2007;30(1):8-17. Chinese.

13. Mapel DW, Dalal AA, Johnson P, Becker L, Hunter AG. A clinical study of COPD severity assessment by primary care physicians and their patients compared with spirometry. Am J Med. 2015;128(6): 629-637.

14. Buffels J, Degryse J, Heyrman J, Decramer M. Office spirometry significantly improves early detection of COPD in general practice: the DIDASCO study. Chest. 2004;125(4):1394-1399.

15. Yu WC, Fu SN, Tai EL, et al. Spirometry is underused in the diagnosis and monitoring of patients with chronic obstructive pulmonary disease (COPD). Int J Chron Obstruct Pulmon Dis. 2013;8:389-395.

16. Lewis K. China's next great leap forward. CMAJ. 2009;180(13): E112-E114.

17. Diggory P, Fernandez C, Humphrey A, Jones V, Murphy M. Comparison of elderly people's technique in using two dry powder inhalers to deliver zanamivir: randomised controlled trial. BMJ. 2001;322(7286): 577-579.

18. Jarvis S, Ind PW, Shiner RJ. Inhaled therapy in elderly COPD patients; time for re-evaluation? Age Ageing. 2007;36(2):213-218.

19. Tan JY, Chen JX, Liu XL, et al. A Meta-Analysis on the impact of disease-specific education programs on health outcomes for patients with chronic obstructive pulmonary disease. Geriatr Nurs. 2012;33(4): 280-296.

20. Monteagudo M, Rodriguez-Blanco T, Llagostera M, et al. Effect of health professional education on outcomes of chronic obstructive pulmonary disease in primary care: a non-randomized clinical trial. Respirology. 2013;18(4):718-727.
21. Shi L. Health care in China: a rural-urban comparison after the socioeconomic reforms. Bull World Health Organ. 1993;71(6): 723-736.

22. Qiu JP, Shu Q, Zhao W, et al. A survey of mastery and application on professional knowledge of health education of the medical staff in some Grade III Level A hospitals in Chongqing City. J Hunan Normal Univ (Med Sci). 2013;2:93-96. Chinese.

23. Weiss BD, Hart G, McGee DL, D'Estelle S. Health status of illiterate adults: relation between literacy and health status among persons with low literacy skills. J Am Board Fam Pract. 1992;5(3):257-264.

24. Fernández-Villar A, López-Campos JL, Represas Represas C, et al. Factors associated with inadequate diagnosis of COPD: On-Sint cohort analysis. Int J Chron Obstruct Pulmon Dis. 2015;10:961-967.

25. Wilson RS, Hebert LE, Scherr PA, Barnes LL, Mendes de Leon CF, Evans DA. Educational attainment and cognitive decline in old age. Neurology. 2009;72(5):460-465.

26. Fan XS, Chen P, Liu ZL. Problems in continuing medical education in grass-roots hospitals and solutions. Chin J Hosp Admin. 2003;19(1): 22-23. Chinese.

27. Feng T, Sun X, Niu W, Wu H, Jiang C. Evaluation of an intervention to improve skills in diagnostic radiology of rural physicians over one year in four rural hospitals. PLoS One. 2014;9(4):e93889.

28. Bordage G, Carlin B, Mazmanian PE. Continuing medical education effect on physician knowledge: effectiveness of continuing medical education: American College of Chest Physicians Evidence-Based Educational Guidelines. Chest. 2009;135(Suppl 3):S29-S36.

29. Ahmed K, Wang TT, Ashrafian H, Layer GT, Darzi A, Athanasiou T. The effectiveness of continuing medical education for specialist recertification. Can Urol Assoc J. 2013;7(7-8):266-272.

30. Hergenroeder AC, Chorley JN, Laufman L, Fetterhoff A. Two educational interventions to improve pediatricians' knowledge and skills in performing ankle and knee physical examinations. Arch Pediatr Adolesc Med. 2002;156(3):225-229.

31. Zhao J, Zhang Z, Guo H, Ren L, Chen S. Development and recent achievements of telemedicine in china. Telemed J E Health. 2010;16(5): 634-638.
International Journal of COPD

\section{Publish your work in this journal}

The International Journal of COPD is an international, peer-reviewed journal of therapeutics and pharmacology focusing on concise rapid reporting of clinical studies and reviews in COPD. Special focus is given to the pathophysiological processes underlying the disease, intervention programs, patient focused education, and self management protocols.

\section{Dovepress}

This journal is indexed on PubMed Central, MedLine and CAS. The manuscript management system is completely online and includes a very quick and fair peer-review system, which is all easy to use. Visit http://www.dovepress.com/testimonials.php to read real quotes from published authors. 